\title{
The Jacquard Machine
}

\author{
By Yoshito Miura, Yoshinosuke Nakajyo and Kunio Suzuki, Members, TMSJ
}

\author{
Kyoto Technological University, Kyoto
}

\begin{abstract}
This article looks into problems which would accompany an attempt to increase the speed of looms equipped with a jacquard machine.

Each lingo vibrates violently with an increase in speed. The grade of its vibrations was judged by the distribution of points of the lingo's descents. As a way to judge that state, sparks were discharged from a lingo placed on special recording paper. A controlled plate for the harness was devised in order to narrow the range of distribution of points of the lingo's descent. A plate was also devised to control the vibrations of the harness. The defective parts resulting from a speed increase were improved, and the warp tension and the weight of the lingo were investigated. The results of these efforts suggest that the speed of a loom equipped with a jacquard machine, at present, $130 \mathrm{rpm}$, can be increased to a maximum of $180 \mathrm{rpm}$.
\end{abstract}

\section{Introduction}

The speed of a loom equipped with a jacquard machine is usually 90 to $130 \mathrm{rpm}$., considerably lower than the speed of a loom without a jacquard machine. It is mainly because of the shedding equipment and the shuttle change equipment, but the shuttle change will not be discussed here.

When the warp is moved by the harness, the lingo which serves as a weight vibrates, thus causing the heald and the warp to vibrate, at times violently enough for the warp to snap. The collision of lingoes scatters metal powder on the fabric. These are some of the impediments to increase the speed. We investigate mainly the lateral vibration of the lingoes.

\section{Loom Speed Limit Imposed by the Distance of the Lingo's Descent}

The warp is lifted as the knife goes up, but is pulled down only by the weight of a lingo. Consequently, the distance of the lingos descent limits the speed of the loom.

Distance $y$, for which the lingo should fall to make a shed while the shuttle is running through the center shed, is given by

$$
y=\frac{b c}{2 a}
$$

where $a$ is the distance between the cloth fell and the forward surface of the shuttle; $b$, the distance between the cloth fell and the counpling or the heald: and $c$, the height of the shuttle.

Assuming that $N \mathrm{rpm}$ is the speed of the loom and $\theta^{\circ}$ the rotational angle of the crank needed to make a whole shed, distance $y$ of the spontaneous descent of the lingo is obtainable as follows:

$$
y=\frac{g}{2}\left(\frac{60}{2 N} \cdot \frac{\theta}{360}\right)
$$

Assuming $a=12 \mathrm{~cm}, b=40 \mathrm{~cm}$ and $c=3 \mathrm{~cm}$, then $y=5 \mathrm{~cm}$ is obtained by formula (1). The values of $N$ obtained by formula (2) where $\theta$ is $324^{\circ}$ and $y$ is over $5 \mathrm{~cm}$ are shown in Table 1 .

Table 1 Relation between the Distance $y$ of Lingo Descent and the Speed $N$ of the Loom

\begin{tabular}{cc}
\hline$y(\mathrm{~cm})$ & $N(\mathrm{rpm})$ \\
\hline 5 & 267 \\
6 & 243 \\
7 & 235 \\
8 & 211 \\
9 & 200 \\
10 & 189 \\
11 & 181 \\
12 & 173 \\
\hline
\end{tabular}

Journal of The Textile Machinery Society of Japan 
Numerous lingoes were used in our experiment, and the distance of the descent of those in the rearmost row was $8 \mathrm{~cm}$. Therefore, the maximum speed was $211 \mathrm{rpm}$, no higher speed being obtainable. Considering the friction between the harness and the comber-board, the friction between harnesses and air resistance, some of the warp presumably remained in the shed.

\section{Method to Measure the Movement of the Lingo}

A lingo being a rod, it is difficult to observe its movement, i.e., its inclination or its displacement from its static position during the assent and descent. Therefore, we judged its lateral movement by the distance between the points of the lingo's descents and its static position. The movement of the lingo was measured with the equipment shown in Fig. 1.

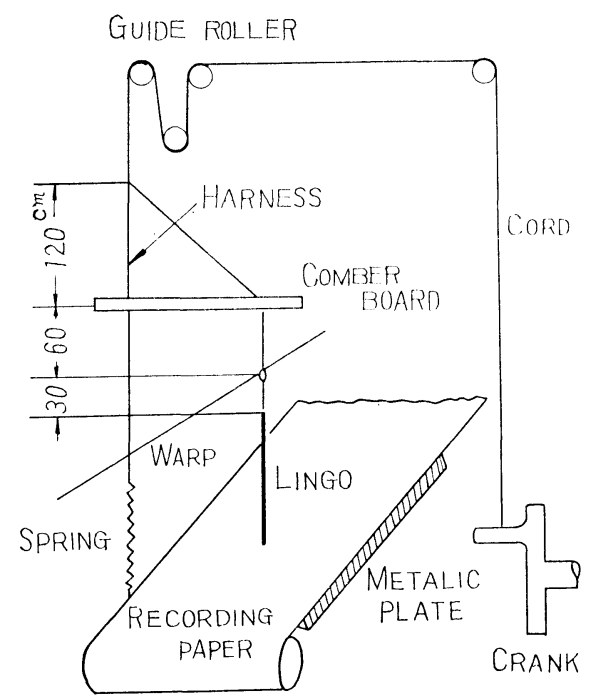

Fig. 1 Equipment to record the descent points of the lingo

The rotating crank actuates the lingo through the guide rollers which control the vibrations of the cord. The proper angular frequency of the spring is $4.36 \mathrm{rad} / \mathrm{sec}$, as follows :

$$
\omega_{e}=\sqrt{\frac{k}{m}}=\sqrt{\frac{0.19}{0.01}}=4.36
$$

where $k$ is the stiffness of the spring and $m$ is its weight.
Assuming that the number of the lingo's ascents and descents is $120 / \mathrm{min}$. then $w_{c}$ is equal to 12.56 $\mathrm{rad} / \mathrm{sec}$. Therefore the vibrations of the spring are scarcely influenced by resonance. In our experiment, the warps were passed through mails placed above the lingo on the loom, so that only the warps were sent out, no weft being used.

The points of the lingo's descents were determined by the following method: Potassium iodide starch paper was mounted on a metallic plate placed under the lingo. Sparks were discharged in the $5 \mathrm{~mm}$ distance between the sharpened tip of the lingo and the paper. As shown in Fig. 2, a current transformer, a rectifier, a condenser and an induction coil were used to generate a highvoltage current, and the sparks were discharged at a suitable rotational angle of the crank only when the lingo fell on the paper.

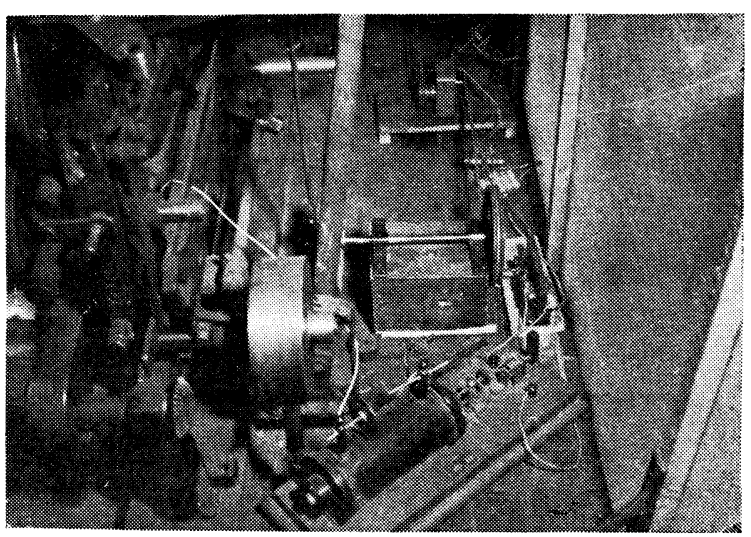

Fig. 2 Discharge equipment

However, as the point of discharge on the recording paper is invisible to the naked eye, the recording paper is then dipped in a mixed solution of cone hydrochloric acid, sodium nitrite and water. If the paper is washed in water immediately, it turns purple except for the points of the spark discharge, which remain white. Mark the points of the spark discharge by making holes there with a pin before drying, because they vanish after drying.

\section{Movement of Individual Lingoes}

The speed of the loom, the weight of the lingo, the angle formed by the harness and the comber. 
board, the warp tension and the size of the shedthese are thought to be the factors which influence the lateral movement of a lingo. Here the speed is fixed at $100 \mathrm{rpm}$ and the other factors are varied as in Table 2.

Table 2 Values of Various Factors

\begin{tabular}{lcc}
\hline \multicolumn{1}{c}{ Factors } & Value & Constant value \\
\hline Weight of the lingo (gr) & $15 \sim 44.2$ & 44.2 \\
\hline $\begin{array}{l}\text { Angle formed by harness } \\
\text { and comber-board (deg) }\end{array}$ & $76 \sim 87$ & 84 \\
\hline Warp tension (gr) & $0 \sim 100$ & 50 \\
\hline Height of shed (cm) & $8 \sim 15$ & 10 \\
\hline
\end{tabular}

The warp is $30 / 2$ 's cotton yarn; the harness, 6-ply twin. The comber-board is No. 2 for cotton weaves (it has holes $2.6 \mathrm{~mm}$ in diameter at intervals of $6.6 \mathrm{~mm}$ in the lateral direction and $5.6 \mathrm{~mm}$ in the longitudinal).
We suspended a lingo from the comber-board and lifted and lowered it for 1 minute. The points of the lingo's descents disperse in the form of an ellipse or a circle. We investigated the distribution of the falling points by drawing concentric circles, all $50 \mathrm{~mm}^{2}$, with the static point of the lingo as the center, and counting the number of the descent points in the ringshaped areas included in the circles. Average of five measurement is shown in Table 3.

Based on the measured values, the influence of the various factors is as follows:

(1) When the descent points disperse within a $10-\mathrm{mm}$ radius, the weight of the lingo should exceed $44.2 \mathrm{gr}$, the angle formed by the harness and the comberboard should exceed $84^{\circ}$, the warp tension being under $50 \mathrm{gr}$ and the height of the shed being less than $10 \mathrm{~cm}$. Constant values in Table 2 are determined from these values.

(2) The range of the distribution of descent points increases as the weight of the lingo and the angle formed by the harness and the comber-board decrease, and as the tension and shed increase.

Table 3 Distribution of Lingo's Descent Points

\begin{tabular}{|c|c|c|c|c|c|c|c|c|c|c|c|c|c|c|}
\hline $\begin{array}{l}\text { Radius of } \\
\text { distribution }\end{array}$ & $(\mathrm{mm})$ & 4 & 5.6 & 6.9 & 8 & 8.9 & 9.8 & 10.6 & 11.3 & 12 & 12.6 & 13.2 & 13.8 & 14.4 \\
\hline \multirow{4}{*}{$\begin{array}{l}\text { Lingo's } \\
\text { weight } \\
\text { (gr) }\end{array}$} & 44.2 & 50 & 19 & 11 & 7 & 6 & 4 & 2 & 1 & & & & & \\
\hline & 28.9 & 51 & 16 & 11 & 8 & 6 & 4 & 2 & 1 & 1 & & & & \\
\hline & 21.1 & 42 & 17 & 12 & 10 & 8 & 5 & 4 & 1 & 1 & & & & \\
\hline & 14.8 & 28 & 16 & 13 & 11 & 8 & 7 & 5 & 4 & 3 & 3 & 1 & 1 & \\
\hline \multirow{5}{*}{$\begin{array}{l}\text { Angle formed } \\
\text { by harness } \\
\text { and board } \\
\text { (deg) }\end{array}$} & 87 & 62 & 18 & 11 & 6 & 2 & 1 & & & & & & & \\
\hline & 84 & 50 & 19 & 11 & 7 & 6 & 4 & 2 & 1 & & & & & \\
\hline & 81 & 37 & 17 & 12 & 8 & 8 & 6 & 5 & 3 & 2 & 2 & & & \\
\hline & 79 & 28 & 16 & 12 & 9 & 7 & 7 & 6 & 5 & 4 & 3 & 2 & 1 & \\
\hline & 76 & 17 & 13 & 11 & 8 & 8 & 7 & 7 & 6 & 6 & 5 & 4 & 4 & 4 \\
\hline \multirow{6}{*}{$\begin{array}{l}\text { Warp } \\
\text { tension } \\
(\mathrm{gr})\end{array}$} & 0 & 69 & 21 & 8 & 2 & & & & & & & & & \\
\hline & 20 & 67 & 15 & 10 & 5 & 2 & 1 & & & & & & & \\
\hline & 40 & 57 & 17 & 12 & 6 & 3 & 3 & 1 & 1 & & & & & \\
\hline & 60 & 53 & 14 & 12 & 8 & 6 & 4 & 2 & 1 & & & & & \\
\hline & 80 & 39 & 15 & 12 & 8 & 7 & 6 & 5 & 4 & 2 & 2 & & & \\
\hline & 100 & 35 & 20 & 14 & 9 & 6 & 6 & 4 & 3 & 2 & 1 & & & \\
\hline \multirow{5}{*}{$\begin{array}{l}\text { Height of } \\
\text { the shed } \\
(\mathrm{cm})\end{array}$} & 8 & 75 & 16 & 7 & 2 & & & & & & & & & \\
\hline & 10 & 50 & 19 & 11 & 7 & 6 & 4 & 2 & 1 & & & & & \\
\hline & 12 & 41 & 15 & 12 & 8 & 7 & 5 & 5 & 4 & 2 & 1 & & & \\
\hline & 14 & 35 & 15 & 12 & 11 & 8 & 7 & 5 & 2 & 2 & 1 & 1 & & \\
\hline & 15 & 27 & 15 & 13 & 10 & 7 & 7 & 6 & 4 & 3 & 3 & 2 & 2 & 1 \\
\hline
\end{tabular}


If the number of descent points should be 100 but the counted number is smaller, it is because points have been duplicated. The larger the range of distribution, the smaller the number of duplications. Duplications occur if measurement takes a long time. In our experiment, duplications were eliminated by reducing the time for measurement to $10 \mathrm{sec}$. Table 3 shows the values obtained in this way. In our experiment, duplicated points were found within $8-\mathrm{mm}$ circle, especially in a $4-\mathrm{mm}$ radius.

\section{Movement of Grouped Lingos and Con- trol of Their Movement}

Lingoes rise and fall collectively on the jacquard machine. Therefore we investigated their lateral group movement (see Fig. 3) by varying their speed.

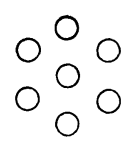

7LINGOES

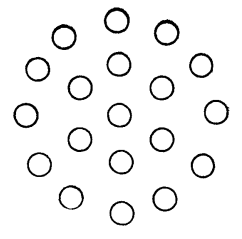

19 LINGOES
Fig. 3 Group of lingos

The investigation was made under the condition in which lingoes make the least movement, i.e., the weight of lingo being $44.2 \mathrm{gr}$; the angle $87^{\circ}$; the tension $0 \mathrm{gr}$ and the height of shed $8 \mathrm{~cm}$.

In a group of seven lingoes, the one in the center was surrounded by the other six lingoes, all the seven being arranged just as the holes were arranged on the comber-board. In the group of 19 lingoes, the one in the center was surrounded by other 18 two fold. Their movements were investigated by two methods:

(1) to let only the center lingo move and keep the others stationary; (2) to keep all lingoes moving.

The lateral movement of the center lingo in either group was measured by coating with lacquer lest its contact with others should cause a short circuit. The speed of lingoes were increased from 30 descents per minute until they vibrated too violent to measure their movement.

A type of equipment different from a jacquard machine was used in this experiment. The grouped lingoes made a motion different from that would be seen on the jacquard machine. So, for com- parison, then, a jacquard machine with a single-lift center shed was attached to a loom and 10 cards were used.

The measurement was made under a condition in which the speed of the loom was $86 \mathrm{rpm}$, the weight of the lingo $21 \mathrm{gr}$, the angle $90^{\circ}$ in the center and $84^{\circ}$ at the end, the stationary tension of the warp $50 \mathrm{gr}$ and the height of the shed $12 \mathrm{~cm}$.

To control the movement of the harness which suspends lingoes, we devised a control board having the same holes as a comber-board. There are two positions (see Fig. 4) to place the control board. One position is above the comber-board. But if it is too high, the angle formed by the position of the control board and the harness will be too small and the friction between them will increase.

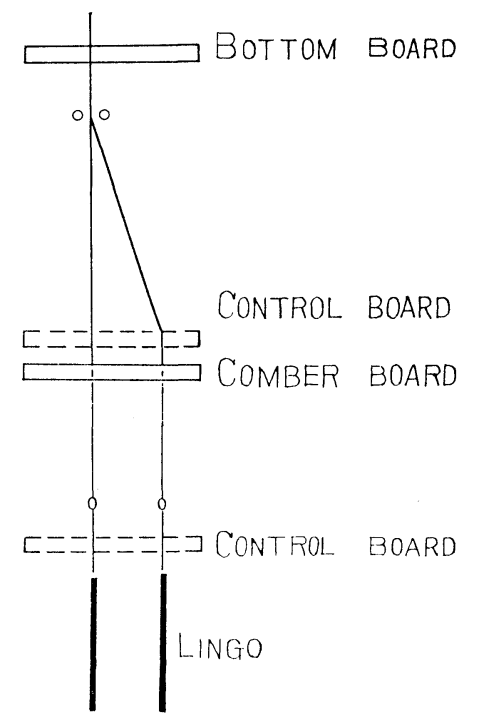

Fig. 4 Position of the control board

The control board should not, therefore, be placed more than $15 \mathrm{~cm}$ above the comber-board.

The other position is between the mail and the lingo. The lower limit of the height of the control board is the tail of the raised-lingo. The breaking strength of the harness was $16 \mathrm{~kg}$ in our experiment and was found to decrease to $12 \mathrm{~kg}$ after the harness worked for one hour at $79^{\circ}$ angle between the control board and the harness. Thereafter it did not decrease even after the harness worked for 10 hours on end.

The maximum speed measurable with and without a control board is shown in Table 4 . This indicates that the limit of the loom speed is 110 to $120 \mathrm{rpm}$., but in this, the lingo vibrates severely. 
Table 4 Maximum and Proper Speed in Case with and without a Control Board

\begin{tabular}{ccccc}
\hline \multirow{2}{*}{ Movement } & \multicolumn{2}{c}{$\begin{array}{c}\text { Max. speed } \\
(\mathrm{rpm})\end{array}$} & \multicolumn{2}{c}{$\begin{array}{c}\text { Proper speed } \\
\text { (rpm) }\end{array}$} \\
\cline { 2 - 5 } & Without & With & Without & With \\
\hline 1 lingo moving & 115 & 125 & 80 & 125 \\
\hline 7 lingoes (1 moving) & 120 & 130 & 100 & 125 \\
(Moving in group) & 110 & 128 & 90 & 125 \\
\hline 19 lingoes (1 moving) & 140 & 158 & 130 & 150 \\
(Moving in group) & 120 & 130 & 95 & 128 \\
\hline
\end{tabular}

As a practical example, when a speed of the loom equipped with a jacquard machine was $86 \mathrm{rpm}$, the radius of the distribution was less than $100 \mathrm{~mm}$. The limit of the speed in this range, without the control board, was $100 \mathrm{rpm}$. It was possible to increase the speed to $120 \mathrm{rpm}$ by using the control board. Now, $120 \mathrm{rpm}$ was the maximum speed of one moving lingo with six stationary. The speed of $120 \mathrm{rpm}$ is obtainable if the radius of distribution is less than $10 \mathrm{~mm}$. A higher speed will be obtainable if weaving is possible in a larger range of distribution.

Now, we investigate the effect of the control board from the density of distribution. The maximum speed is the sum total of the distribution density of the first ring ( $4 \mathrm{~mm}$ in radius) and the second ring ( $6 \mathrm{~mm}$ in radius), and is 1.1 to 1.2 . It amounts to half the total density $(=2)$ of all rings. The proper speed is obtainable if the density of the first ring exceeds 1 and the total density of the first and second rings equals 1.4 to 1.5. Use of the control board increases the maximum speed by $10 \mathrm{rpm}$ and the proper speed by $20 \mathrm{rpm}$ at least.

\section{Adjusting the Jacquard Machine}

Fig. 5 shows the neighbourhood of the cylinder. With $l$, the horizontal distance from the cylinder to the roller made longer than $l_{1}$, the horizontal distance from the cylinder to the end of the card cradle, the card on the cradle is lifted diagonally. The moment the card drops out of the card cradle, the binding cards vibrate. Therefore, $l_{1}$, should be made longer than $l_{1}$, used in our experiment was $90 \mathrm{~cm}$. With $l$ exceeding $60 \mathrm{~cm}$, the cards between the cylinder and the roller vibrate severely. $l$ should, therefore,

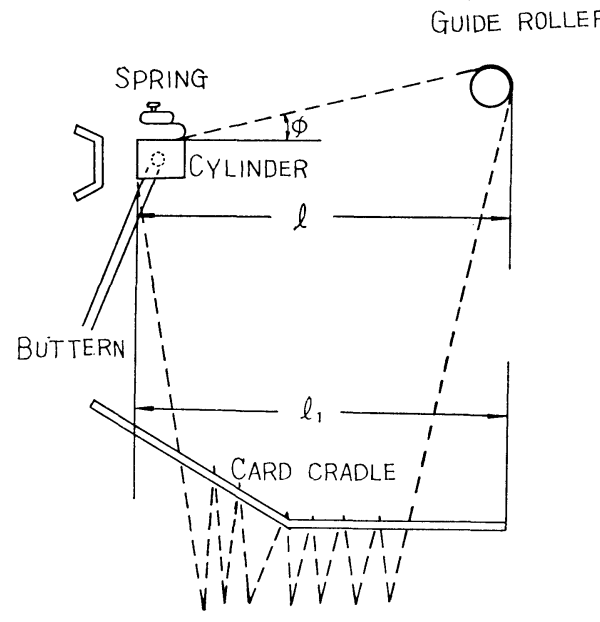

Fig. 5 The neighbourhood of the cylinder

properly b: 40 to $60 \mathrm{~cm}$.

Fix $l$ at $50 \mathrm{~cm}$ and vary the height of the roller. If the angle $\phi$ formed by the roller and the horizontal line is below $7^{\circ}$, the jacquard machine is capable of normal operation at a speed not above $180 \mathrm{rpm}$. With $\phi$ over $7^{\circ}$, the card leaves the cylinder. If the swing distance $e$ of the buttern is shorter than $5 \mathrm{~cm}$, the cylinder does not vibrate. If it is longer than $8 \mathrm{~cm}$, both the cylinder and the catch vibrate severely. The motion is repeated at regular intervals; the larger $e$, the higher the speed of the buttern. The result is that the catch which revolves the cylinder and even the cylinder vibrate.

Consider the operation of the spring which presses the card against the cylinder. If the number of cards is as small as 48, they are strained between the cylinder and the roller. At this time, even if the machine is run at $180 \mathrm{rpm}$ without a spring, the card does not get off the cylinder. If as many as, say, 240 cards are used, they get off unless a spring is used. If the spring is used, they do not get off even if the machine runs at $180 \mathrm{rpm}$. Use of a spring, therefore, increases the speed of the jacquard machine.

\section{Warp Tension}

Now we investigate the shedding in the center and the edge of a group of warps. On the edge of the weaving width, the comber-board was shifted about $22 \mathrm{~cm}$ from the center. With the speed $170 \mathrm{rpm}$ in the center shedding and above $160 \mathrm{rpm}$ 
in the center shedding and above $160 \mathrm{rpm}$ in the edge shedding, the cotton yarns vibrated severely and the shed was not uniform. The reason is presumably that some of the warps forming the lower shed were lifted by the warps which formed the upper shed. So, we tried to increase the warp tension. When the stationary tension of the warp was above $50 \mathrm{gr}$ in the middle and above $70 \mathrm{gr}$ on the edge, the shedding condition was good with $180 \mathrm{rpm}$. The vibration problem can be solved by making all the warp tensions about $70 \mathrm{gr}$. The distribution of the descent points of the lingo shows that the smaller the warp tension, the better. With the warp tension below $60 \mathrm{gr}$, the distribution was much poorer than that with the tension above $80 \mathrm{gr}$. A tension of $70 \mathrm{gr}$. is advisable.

\section{Results of Driving Test}

By making various adjustments suggested, the speed of the loom with the jacquard machine was varied from $100 \mathrm{rpm}$ to $200 \mathrm{rpm}$. It was found that the problems accompanying an attempt to increase the speed over $160 \mathrm{rpm}$ and measures to cope with them were as follows:

(1) The screws and the nuts in every part loosen if the speed is increased.

(2) It is not done effectively to control the raising of warps by the punched cards. If the distance between the top end of the knife and the top end of the hook when the knife has made the lowest descent increases, the knife and even unnecessary hooks go up. The best distance is $7 \mathrm{~mm}$. The proper distance between the hook pushed and the knife is $9 \mathrm{~mm}$.

(3) The cylinder catch cannot drive the cylinder accurately at high speed. The remedy is to make a hole in the head of the catch to pass the spring through it.

(4) The lingo should be heavy to suppress its vibration. The vertical movement of the harness is easy even at $180 \mathrm{rpm}$ if the harness enters the comber-board vertically. When $\gamma$ is the angle formed by the harness and the comber-board, and $M$ is the weight of the lingo, the frictional force is $\mu M \cos \gamma$. Assuming that the coefficient of friction is 0.3 and the maximum of $\gamma$ is $76^{\circ}$, the force $F$ of the descent can be given by

$$
F=M(1-0.0754)
$$

$F$ on the edge is $8 \%$ less than $F$ in the center. To keep $F$ constant, $M$ has to be large in proportion to its distance from the center, but it is easier said than done. One alternative is to make $M$ heavy. For $F$ on the edge close in value to $F$ in the center, it is enough to make $M$ equal $20.6 \mathrm{gr}$. We have said that the proper weight of the lingo is above 42.2 gr, but a lingo over 20 gr makes a good distribution of the descent-points, as shown in Table 3. Therefore, $20.6 \mathrm{gr}$ is all right as the weight of a lingo.

\section{Conclusions}

If a loom equipped with a jacquard machine is increased in speed over $16^{\circ} \mathrm{rpm}$, the screws, bolts, and nuts of the jacquard machine loosen, the cylinder catch comes off the cylinder, and the warp does not move according to the directions given by the cards. This problem can be solved by adjusting the jacquard machine and making the screws, bolts and nuts precisely.

The vibrations of the lingo can be observed by recording the points of its descents by discharging sparks from the tip of the descending lingo to special recording paper. The range of the distribution of descent points is influenced by the speed, the weight of the lingo, the warp tension, the angle formed by the harness and the comber-board, the descent distance of the lingo, etc.

Lingo vibrations can be controlled by restricting the movement of the harness with the aid of the frame which encloses the lingoes; and the control board through which the harness passes.

To make the warp lines uniform, the weight of the lingo and the tension of warp should be adjusted. For example, when cotton yarns 30/2's are woven with a jacquard machine using a single-lift center shed, the loom can be increased in speed to $180 \mathrm{rpm}$, by making the weight of the lingo $20.6 \mathrm{gr}$, the static tension of warp $70 \mathrm{gr}$, and placing the control board (which is the same in build as the comber-board) $15 \mathrm{~cm}$ above the comber-board. 